\title{
Reflets
}

Revue ontaroise d'intervention sociale et communautaire

\section{La spiritualité : au coeur ou en marge de l'intervention sociale?}

\section{Nérée St-Amand}

Volume 12, numéro 1, 2006

Spiritualité et intervention sociale

URI : https://id.erudit.org/iderudit/013437ar

DOI : https://doi.org/10.7202/013437ar

Aller au sommaire du numéro

Éditeur(s)

Reflets : Revue ontaroise d'intervention sociale et communautaire

ISSN

1203-4576 (imprimé)

1712-8498 (numérique)

Découvrir la revue

Citer cet article

St-Amand, N. (2006). La spiritualité : au coeur ou en marge de l'intervention sociale? Reflets, 12(1), 20-47. https://doi.org/10.7202/013437ar

Tous droits réservés (C) Reflets : Revue ontaroise d'intervention sociale et communautaire, 2006
Ce document est protégé par la loi sur le droit d'auteur. L'utilisation des services d'Érudit (y compris la reproduction) est assujettie à sa politique d'utilisation que vous pouvez consulter en ligne.

https://apropos.erudit.org/fr/usagers/politique-dutilisation/ 


\section{La spiritualité : au cœur ou en marge de l'intervention sociale?}

Nérée St-Amand,

Professeur, École de service social, Université d'Ottawa

L'homme moderne ne comprend pas à quel point son rationalisme, qui a détruit sa faculté de réagir à des symboles et à des idées, l'a mis à la merci de ce monde psychique souterrain. Il s'est libéré de la "superstition " (du moins, il le croit) mais ce faisant, il a perdu ses valeurs spirituelles à un degré alarmant. Ses traditions morales et spirituelles se sont désintégrées, et il paie cet effondrement d'un désarroi et d'une dissociation qui sévissent dans le monde entier.

C. G. Jung 1964 : 82 et 94

\section{Au XXI ${ }^{e}$ siècle, oser parler de spiritualité en travail social?}

Depuis le Siècle des lumières, l'Occident s'est tourné vers la science pour résoudre ses problèmes les plus complexes, expliquer l'évolution du monde, conquérir l'espace, guérir les gens. Dans le domaine des communications interpersonnelles et dans celui de la psychothérapie, le même phénomène peut être observé : la confiance en la science, une science basée sur le rationnel,le mental, l'intellect, s'est instaurée et a supplanté des traditions millénaires 
"La suprématie scientifique que nous observons depuis un siècle en particulier fait en sorte que des concepts tels la compassion, la solidarité, le pardon, la conscience sociale, la transcendance, se trouvent marginalisés dans la littérature et dans les pratiques des sociétés basées sur les impératifs $d u$ marché. " établies sur des valeurs, pratiques et principes tels l'intuition, la télépathie, le rêve ou l'astrologie, pratiques qui sont d'ailleurs mieux acceptées de nos jours dans les sociétés traditionnelles et les milieux populaires que par le monde intellectuel, scientifique, universitaire. C'est ainsi que se sont développées, au cours de l'époque dite moderne, des théories explicatives de l'univers et, en contrepartie, des façons de penser et d'intervenir qui sont témoins de cette logique de la suprématie de la science et de l'intellect. De telle sorte que, de nos jours, la très grande majorité des disciplines proposent des théories ou interprétations des faits basées sur l'observable, le quantifiable, l'analyse, la raison.

Or, la spiritualité ne fait à peu près pas partie de cet univers, de cette vision des choses, sans doute parce que non mesurable selon les principes de cette science ou, tout simplement, parce que considérée par d'aucuns comme dépassée. La suprématie scientifique que nous observons depuis un siècle en particulier fait en sorte que des concepts tels la compassion, la solidarité, le pardon, la conscience sociale, la transcendance, se trouvent marginalisés dans la littérature et dans les pratiques des sociétés basées sur les impératifs du marché. Pourtant, certains scientistes de renom, Einstein entre autres, nous ont prévenus des limites d'une science basée sur l'intellect humain :

"We should take care not to make intellect our god. It has, of course, powerful muscles, but no personality. It cannot rule, only serve. "

http://www.quotationspage.com/quotes/Albert_Einstein/

Dans la foulée de ce mouvement de primauté intellectuelle, d'aucuns prétendent que la théorie darwinienne de l'évolution aura consolidé une rupture importante entre le spirituel et l'intellectuel (Coquin : 48), puisqu'elle a instauré une science où non seulement prime la raison, mais d'où, par ricochet, le sacré est évacué.

Plus près de nous, sur le plan des professions vouées à l'aide, Freud s'est imposé comme celui qui pouvait expliquer les causes profondes de nos malaises et de nos problèmes. Bien que Gustav 
"...nous nous

retrouvons aujourd'hui avec un foisonnement de théories fondées sur un modèle palliatif de l'aide, limité à sa dimension logique, mesurable, d'où est évacué le volet spirituel."
Jung, un penseur beaucoup plus spirituel, se soit fortement dissocié de l'analyse proposée par son collègue et ami d'un moment, les fondements de la psychologie, de la psychanalyse et de la psychiatrie modernes ont davantage retenu les théories freudiennes que celles proposées par Jung. Ce dernier insistait, entre autres, sur l'importance de la dimension spirituelle de l'être humain (Nash : 135). En effet, à l'opposé de Freud pour qui à peu près tout tournait autour du rêve et de la sexualité, Jung osa parler de "la métamorphose de l'âme» (1976). Son intérêt pour les cultures et l'histoire, son esprit ouvert à la dimension spirituelle des problèmes de l'existence (Jung 1998) ont fait de lui une personnalité de premier plan en ce qui a trait à l'analyse holistique, bien plus que Freud dont l'analyse était tout aussi pointue que basée sur des principes observables.

Qui plus est, l'analyse des problèmes sociaux a vite fait d'adopter, au $\mathrm{XX}^{\mathrm{e}}$ siècle en particulier, un raisonnement classique, basé sur les enseignements de Freud et sur des approches à caractère médical, de sorte que nous nous retrouvons aujourd'hui avec un foisonnement de théories fondées sur un modèle palliatif de l'aide, limité à sa dimension logique, mesurable, d'où est évacué le volet spirituel. Mises à part quelques approches structurelles, peu de théories du social se montrent intéressées aux dimensions globales des problèmes auxquels nous faisons face (pauvreté, violence, inégalités, misère, oppression). Cela s'est produit en dépit de la vision de l'une des deux fondatrices de notre profession, Jane Addams, qui proposait une analyse de fond des problèmes que nous tentons de résoudre, basée sur la responsabilité sociale, donc sur une conscience des enjeux éthiques de l'intervention :

Jane Addams' writings and activities at Hull House reveal an ethic that revolved not around religious belief, but around action that served to better the community. She incorporated her vague, pragmatic spirituality into a social ethic that was ultimately secular; an ethic built on a foundation of democracy rather than religion (McKoy 2005). 
"Malgré le fait qu'elle fut la seule travailleuse sociale récipiendaire du prix Nobel de la paix, Addams est loin d'avoir le crédit dont jouit aujourd'hui Mary Richmond."
En psychologie, Jung (1976) appréhendait la réalité de façon globale; Addams (2005) a fait de même en service social. Or, elle a hérité d'un sort encore plus marginal que celui réservé à Jung. Malgré le fait qu'elle fut la seule travailleuse sociale récipiendaire du prix Nobel de la paix, Addams est loin d'avoir le crédit dont jouit aujourd'hui Mary Richmond. Preuve de plus que l'histoire est sélective dans les personnes qu'elle retient et que l'idéologie holistique à laquelle adhéraient les visionnaires du début du $\mathrm{XX}^{\mathrm{e}}$ siècle explique peut-être notre tendance à les oublier.

Par ailleurs, beaucoup de personnes, travaillant principalement dans le domaine des alternatives, en intervention sociale ou ailleurs, estiment que nos grands problèmes sociaux ne seront jamais résolus tant que nous nous centrerons sur des approches professionnelles, scientifiques, à la Freud ou à la Richmond, qui ne tiennent pas compte de la dimension sacrée, de la dimension spirituelle (Canda, Boiron, Platteau). L'objectif de cet article est justement de tenter une percée dans ce monde largement inexploré de la spiritualité dans l'intervention sociale et d'en proposer quelques pistes de réflexion applicables aux professions dites aidantes.

\section{Une percée sur le plan de la spiritualité : le Forum de Barcelone}

Au cours de l'été 2004,j'ai eu la chance de participer au Forum de Barcelone, cet immense rassemblement de milliers de conférenciers venus des quatre coins de la planète et dont les propos étaient axés sur le développement durable, une conscience sociale globale, un partage équitable des ressources de la planète et des préoccupations de justice sociale, voire de survie de l'humanité. Pendant plus de quatre mois, sur le thème du Dialogue des cultures, ce forum a été témoin de personnes proposant de penser autrement, d'axer le développement sur l'humain et sa relation avec la Terre, de reconnaitre l'art comme forme d'expression politique, d'affermir la spiritualité et la culture comme étant au cœur de la survie 
"...plus de 8000 personnes étaient venues réfléchir sur le vide spirituel de nos sociétés modernes, mais aussi sur la transition qui est en train de s'effectuer entre un univers laïc... et un monde où le spirituel est ancré au cour du quotidien,...» de notre civilisation. C'est ainsi que nous avons vu défiler, dans un décor des plus enchanteurs à la Gaudi, des femmes et des hommes, chercheurs, intervenants, artistes et créateurs de toutes provenances, venus exprimer leur foi en un monde centré sur des valeurs d'altérité, de conscience, d'engagement, de spiritualité. Une cinquantaine de forums, d'une durée de cinq à huit jours chacun, foisonnant d'activités artistiques, culturelles et spirituelles, ont ainsi constitué une première, à l'échelle mondiale, en écho au sommet de Porto Alegre, qui lui était axé de façon plus pointue sur l'écologie.

Dans le forum où j'ai participé, le Parlement des religions $d u$ monde, plus de 8000 personnes étaient venues réfléchir sur le vide spirituel de nos sociétés modernes, mais aussi sur la transition qui est en train de s'effectuer entre un univers laï — principalement occidental et qualifié de moderne - et un monde où le spirituel est ancré au cœur du quotidien, aux valeurs empruntées surtout de l'Orient, mais également aux cultures traditionnelles et aux sociétés autochtones. Au cours de ces huit jours, plus de 500 présentations ont exploré le rôle de la spiritualité dans le monde moderne, ses diverses manifestations et dimensions morales, éthiques, sociales, en se basant sur une pléiade d'auteurs, de maîtres, de guides ou de productions audio-visuelles (http://www.cpwr. org/2004Parliament/).

Il faut dire que cette grande conférence s'était ouverte d'une façon fort originale : par une méditation silencieuse. En effet, avant toute cérémonie d'usage, la parole a laissé la place au silence, un fait inusité dans les annales scientifiques modernes. Et l'auteur de cette intervention, Sri Chinmoy, maitre spirituel en provenance de l'Inde, croit justement que le silence est un outil plus fort, plus intense que la parole (Sri Chinmoy 2002). De quoi remettre en question plusieurs présupposés scientifiques basés sur la parole et sur l'écrit, dans une société qui semble avoir oublié la place relative des mots en liens avec les autres formes que peuvent prendre des vérités non traditionnelles: «Spirituality has taught me the difference between my speech and my silence, between my mind and my heart. In speech I try to become. In silence I am.» (Sri Chinmoy $1972:$ 1). 
En m'inspirant de cette conférence et de mes pratiques d'intervention, la suite de cet article se veut une réflexion basée sur mes expériences en tant qu'intervenant et qu'enseignant en service social, mais aussi en tant que personne tentant de pratiquer une forme de spiritualité dans un monde où priment les valeurs d'avoir, de consommation, de possessions. Ce texte se veut ainsi une ouverture à d'autres dimensions du monde et à de nombreux groupes de conscientisation qui aspirent à des valeurs d'altérité, de partage, de transcendance et qui, pour ce faire, privilégient une vision spirituelle de l'Univers, de l'Autre, de notre passage sur terre.

J'essaierai d'explorer quelques aspects possibles de l'intervention sociale lorsque celle-ci est ancrée dans la spiritualité, dans le sacré. Je me propose également de présenter diverses composantes d'un renouvellement des pratiques d'intervention actuelles en me basant sur certains principes et quelques auteurs qui m'ont inspiré dans cette démarche.

\section{Religion ou spiritualité?}

Au début du $\mathrm{XX}^{\mathrm{e}}$ siècle, Sri Aurobindo avait déjà proposé une distinction marquante entre religion et spiritualité; il définissait la religion telle qu'elle a évolué jusqu'ici comme étant intellectuelle, basée sur des valeurs sociales comme le service, le respect, et ancrée principalement sur des valeurs de foi, d'adoration. Une telle religion basée sur des dogmes, des rites, des valeurs de charité, constitue un mouvement préparatoire de purification vers des transformations spirituelles; c'est ainsi que nous évoluons actuellement vers ce qu'il appelle la religion spirituelle de l'humanité, qu'il décrit ainsi :

Une religion spirituelle de l'humanité suppose la perception grandissante qu'il existe un Esprit secret, une Réalité divine en laquelle nous sommes tous un, que l'humanité est à présent sur la terre son plus haut 
«Pour Lemieux

...la spiritualité, ...se distancie d'une conception traditionnelle de la religion dans le sens où une marge grandissante est en train de s'établir entre les deux univers..."

"...un nombre grandissant de personnes, critiques face aux discours opprimants des grandes religions, restent en quête de sens, de sacré. " véhicule, et que le genre humain et l'être humain sont les moyens par lesquels cette Réalité se révélera progressivement ici-bas. Elle implique un effort grandissant pour vivre cette connaissance et instaurer sur la terre le royaume de cet Esprit divin (1999 : 452).

Pour Lemieux par ailleurs, la spiritualité, telle que nous l'abordons ici, ou religion spirituelle comme la décrit Sri Aurobindo, se distancie d'une conception traditionnelle de la religion dans le sens où une marge grandissante est en train de s'établir entre les deux univers :

Serions-nous dès lors en présence d'une sorte de disjonction entre l'univers religieux, associé aux institutions, et l'univers spirituel, associé à la liberté des sujets? Sevré de religion, le monde contemporain serait-il en train de réinventer la spiritualité? (Lemieux : 2)

En effet, un nombre grandissant de personnes, critiques face aux discours opprimants des grandes religions, restent en quête de sens, de sacré. Pour ce faire, ces gens se tournent vers des approches à caractère spirituel inspirées principalement de l'Orient et de l'hémisphère sud (Inde et Afrique, en particulier). Dans un Occident qui a été brimé ou même parfois piégé par les grandes religions, où l'expression de soi a souvent dû être sanctionnée par des institutions religieuses, notre réaction face à une spiritualité grandissante est à la fois de fascination et de crainte (Lemieux : 2). Fascination de voir tellement de manifestations d'une spiritualité aux dimensions multiples ${ }^{1}$. En effet, le spirituel engage la vie, profondément, alors que le religieux reste plutôt réservé à une relation à Dieu, grâce surtout à l'intermédiaire de ses représentants, et basé en Occident sur les grandes religions. Double réaction donc, d'envoûtement, mais aussi de méfiance face à un spirituel renaissant, car nous ne voulons surtout pas nous faire "re-prendre " à certains pièges de la religion, comme les générations qui nous ont précédés, ni par certains représentants de Dieu qui avaient comme préoccupation la satisfaction de leurs propres besoins plutôt que des voies divines ${ }^{2}$. Reste tout de même 
"La spiritualité peut alors être comprise comme cette recherche de sens, de connexions, d'appartenance à quelque chose de plus grand, de plus profond que soi." que ces religions ont apporté au monde des grands mouvements de transformation qui ont grandement affecté l'histoire et les civilisations, que l'on pense, par exemple, au Soufisme dans l'Islam ou aux grandes écoles inspirées de Saint François d'Assise.

La spiritualité peut alors être comprise comme cette recherche de sens, de connexions, d'appartenance à quelque chose de plus grand, de plus profond que soi. Ainsi, les rapports à la terre, à l'héritage, aux ancêtres, au monde des esprits, la promotion de l'harmonie, de l'équilibre intérieur, et entre personnes et communautés, sont considérés au cœur de la spiritualité (Canda 1988 : 33-34). Sri Chinmoy définit cette recherche comme l'écoute de la voix intérieure, celle qui nous permet d'éviter les illusions du monde extérieur pour nous centrer sur des recherches d'absolu; elle est notre richesse, notre appel à la transcendance (1972: 45).

\section{Spiritualité et service social : l'urgence d'un changement de cap}

We are all children of the Great Spirit; we all belong to Mother Earth. Our planet is in great trouble and if we keep carrying old grudges and do not work together, we will all die. Chief Seattle, http://www.galafilm.com/1812/e/people/tec_speach2.html

Plusieurs personnes et mouvements décrient les préjugés et les limites des approches psychologisantes, individualisantes, qui sont actuellement à la base des professions d'aide telles que nous les connaissons aujourd'hui (Platteau, St-Amand 2001). Par contre, les pratiques en émergence que d'aucuns proposent (Sivaraska, Nash, St-Amand 2003) visent à revitaliser la société, à lui redonner des valeurs éthiques, une composante spirituelle, pour que s'affirment l'ailleurs et l'autrement. De telles approches se manifestent grâce à d'autres façons de voir et de faire, basées sur l'harmonie, la transcendance, la générosité. Plusieurs parlent de l'éveil d'une nouvelle conscience, d'un voyage intérieur, d'effacement de l'ego 
"Plusieurs parlent de l'éveil d'une nouvelle conscience, d'un voyage intérieur, d'effacement de l'ego..., alors que d'autres témoignent d'une transformation radicale de l'ordre actuel des choses, d'une contre-civilisation et d'une redéfinition du concept de développement. »
(Abot: 7), alors que d'autres témoignent d'une transformation radicale de l'ordre actuel des choses, d'une contre-civilisation et d'une redéfinition du concept de développement.

The death and destruction throughout the world today compel us to think and act together to overcome all suffering and bring about the awakening of humankind. To alleviate suffering, we must always go back to our own spiritual depths, to retreat, meditation, and prayer. It is nearly impossible to sustain the work otherwise (Sivaraska : 116).

Certes, d'autres valeurs sont en train de poindre, ou de "re-naitre» peut-être, valeurs oubliées temporairement par un monde matériel assoiffé d'avoirs. Plusieurs nous ont mis en garde contre cette pauvreté intérieure ou spirituelle (Sri Chinmoy 1972 : 77) que l'Occident a amenée avec sa vision matérielle du développement, du progrès; d'autres nous avertissent de l'impasse qui nous guette si nous continuons dans cette direction :

A great gulf has been opened between man's material advance and his social and moral progress, a gulf in which he may one day be lost if it is not closed or narrowed (Lester B. Pearson, Discours lors de la réception du prix Nobel de la paix, 1957).

\section{«Un maillon dans une chaîne ": mondialisation des solidarités}

A human being is a part of the whole called by us "the universe", a part limited in time and space. He experiences himself, his thoughts and feelings, as something separate from the rest, a kind of optical delusion of his consciousness. This delusion is a kind of prison for us, restricting us to our personal desires 
and affection for a few persons nearest to us. Our task must be to free ourselves from this prison by widening the circle of understanding and compassion to embrace all living creatures and the whole of nature in its beauty (Albert Einstein: http://www.heartquotes.net/ consciousness.html).

La spiritualité évolue de plusieurs façons et selon plusieurs principes. En fait, ses manifestations sont fort variées, éclatées même, et touchent de nombreuses sphères de la société, que ce soit en science, en santé, en éducation et, pratiquement, en tout ce qui concerne la vie quotidienne. Mais c'est généralement le concept de Oneness, d'unité, qui s'en dégage comme dénominateur commun.

Only when one is totally united with the rest of the world can he-she truly be happy. And this happiness makes a person the unparalleled soul on earth. It is not power that makes us superior or makes us feel that we are priceless; it is our matchless oneness with the Absolute. Others do not need us because we have power. No, others badly need our soul's oneness (Sri Chinmoy 1972 : 48-49).

"Le terme

"mondialisation des

solidarités " exprime

bien cette préoccupation

universelle $d u$

bien commun que

Sri Aurobindo, ce philosophe, poète, révolutionnaire, avait bien identifiée au début du $X X^{e}$ siècle..."
Nous ne formons qu'un; nos diversités, nos divisions ne doivent pas nous distraire de notre but commun et collectif. Le terme "mondialisation des solidarités " exprime bien cette préoccupation universelle du bien commun que Sri Aurobindo, ce philosophe, poète, révolutionnaire, avait bien identifiée au début du $\mathrm{XX}^{\mathrm{e}}$ siècle :

Les individus qui aideront le plus l'avenir de l'humanité en cet âge nouveau seront ceux qui reconnaîtront qu'une évolution spirituelle est la destinée de l'être humain, et donc son besoin le plus profond. De même que l'homme animal s'est largement converti à une humanité mentalisée, et même hautement mentalisée à son sommet, de même le type actuel 
d'humanité doit maintenant, ou dans l'avenir, évoluer ou se convertir à une humanité spiritualisée, car tel est le besoin de l'espèce et certainement l'intention de la nature (1994: 411).

\section{Conséquences pour le service social}

En revoyant quelques-uns des principes sur lesquels se base la spiritualité, je tenterai de proposer certaines conséquences pour le service social, si nous acceptons de pratiquer l'intervention sociale de façon holistique, tenant compte des valeurs de transcendance que propose une telle vision de l'univers et de la place que pourrait y occuper notre profession.

\section{Premier principe : 1 'intervention sociale sur fond de spiritualité}

Dans ma formation à l'intervention sociale, jamais je n'ai eu de maîtres, jamais on ne m'a présenté d'écrits qui m'aient invité à réfléchir à la dimension spirituelle dans l'intervention sociale ou qui aient même abordé le concept de spiritualité. Les grands auteurs qui ont articulé l'intervention sociale au $\mathrm{XX}^{\mathrm{e}}$ siècle ont adhéré à des approches psychologisantes ou structurelles et sont passés à côté des dimensions spirituelles de l'aide ${ }^{3}$. Tout se passe comme s'ils n'étaient pas à l'aise lorsqu'il s'agit d'aborder un espace qui les dépasse. Ou encore, se peut-il qu'ils considèrent la spiritualité comme non pertinente à la relation d'aide? À l'instar de la science, les grands théoriciens de l'intervention tentent de tout expliquer, de tout disséquer, selon des approches basées sur la division logique du processus de l'intervention. Même les tenants des approches d'empowerment, qui prônent pourtant le pouvoir d'agir citoyen et qui veulent que la prise en charge revienne totalement aux personnes et aux collectivités défavorisées et démunies, n'abordent à peu près jamais la dimension spirituelle de celles et de ceux qu'ils veulent respecter dans leur altérité 
«Les sociétés

autochtones par ailleurs, ... abordent systématiquement et en tout premier lieu la spiritualité, la relation entre l'humain et les réalités intemporelles, le sacré, et ce, dans toutes leurs interventions comme dans tous leurs écrits,... »
(Dufort et Guay 2001, Breton 2002). Dans le domaine de l'aide, Jung constitue une remarquable exception à cette observation; le service social aurait intérêt à revenir à ses écrits. Sur le plan scientifique, Einstein a eu le courage, sinon l'audace, d'admettre les limites de la science basée sur la raison.

My religion consists of a humble admiration of the illimitable superior spirit who reveals himself in the slight details we are able to perceive with our frail and feeble mind (Albert Einstein http://www.quatationspage. com/quotes/Albert_Einstein).

Les sociétés autochtones par ailleurs, celles qui ont su préserver leurs cultures millénaires, abordent systématiquement et en tout premier lieu la spiritualité, la relation entre l'humain et les réalités intemporelles, le sacré, et ce, dans toutes leurs interventions comme dans tous leurs écrits, tel que le suggère cette prière amérindienne :

May the great Spirit grant you the strength of Eagle's Wings; the Faith and Courage to fly to new Heights, and the Wisdom to rely on His Spirit to carry you there (http://thespiritofthewolf.tripod.com/id3.html).

C'est chez les sages autochtones que j'ai d'abord pu puiser une inspiration et des exemples de pratiques où la spiritualité se situe au cœur du quotidien (Brown, Bourgeault, The Sacred Tree). Fort de ces exemples, je me suis tourné vers d'autres auteurs qui tout simplement éliminent les murs entre deux formes de compréhension, sur fond d'harmonie, d'unité, de conscience : Sivarasta, Sri Chinmoy, Sri Aurobindo, Carlos Castaneda, Khalil Gibran, St-Exupéry, Jean Vanier; ceux-ci ont inspiré mon quotidien et guidé mes réflexions, axées sur l'intervention sociale à caractère spirituel.

\section{Deuxième principe : voir avec le cœur. «L'essentiel est invisible pour les yeux "}

Pour les amateurs des approches alternatives, St-Exupéry a légué une merveilleuse histoire remplie de simplicité et de sagesse. Celle 
"De nos jours, beaucoup interviennent selon un regard et des principes proches de ceux présentés dans Le Petit Prince.»
"Notre jugement de l'autre dans sa différence, dans sa déviance, pourrait être tout simplement éliminé si nous réussissions à le voir avec un regard spirituel. » d'un petit prince qui voit autrement, qui exprime des réalités à la fois belles et pures, basées sur une analyse tout aussi candide que spirituelle de notre raison d'exister. De nos jours, beaucoup interviennent selon un regard et des principes proches de ceux présentés dans Le Petit Prince. On peut penser à Jean Vanier (Spink 1990) par exemple, qui a vu le Divin dans le regard d'une personne que notre monde qualifiait de "retardé mental ». Dès lors, il a abandonné son poste de professeur de philosophie pour consacrer sa vie à établir des communautés de l'Arche, basées sur le partage, une façon spirituelle de voir les personnes avec un handicap intellectuel, marginalisées, exclues sur la base de leur incapacité de produire ou de s'insérer dans notre société marchande. Depuis, plus de 400 maisons de l'Arche, dans 30 pays, accueillent des personnes handicapées intellectuellement, mais dont l'évolution spirituelle est marquante. On n'a qu'à visiter une de ces maisons pour sentir que la personne y est au cœur et que le sourire des résidents vaut davantage que leur soi-disant quotient intellectuel.

Autre exemple, sur un tout autre plan cette fois : lors de mes voyages en Orient, je suis toujours frappé, entre autres, par la densité, voire l'intensité, de la circulation routière. Piétons, bicyclettes, mobylettes, motocyclettes, voitures, camions, autobus se croisent et s'entrecoupent, à quelques centimètres près. Qui plus est, dans un tel décor, certaines gens circulent, à motocyclette ou à bicyclette surtout, en sens opposé de cette circulation très dense. Personne ne semble s'en plaindre, personne ne semble même en faire de cas. Lorsque j'en ai eu l'occasion,j'ai demandé à un guide local comment de telles pratiques pouvaient s'expliquer, voire être tolérées. Il me répondit tout simplement : " S'ils circulent en sens opposé, c'est qu'ils ont une raison de le faire! » De retour chez moi, je me suis dit que si nous appliquions aux comportements humains " déviants » ce civisme oriental, beaucoup de problèmes seraient tout simplement éliminés. Finis en très grande partie le Diagnostic Statistical Manual (DSM), les diagnostics de troubles de comportement, les évaluations d'hyperactivité chez les enfants, finie la délinquance juvénile et ses comportements déviants... Notre jugement de l'autre dans sa différence, dans sa déviance, pourrait être tout simplement éliminé si nous réussissions à le voir 
"Si nous nous inspirions de Jane Addams, nous constaterions que le problème ne se situe pas sur le plan de la personne qui vit dans sa peau les contradictions de notre société marchande. » avec un regard spirituel. Si telle personne est là, si elle pense ou agit comme ça, c'est qu'elle a ses raisons, c'est que pour elle, penser ou agir ainsi est logique. Donc, respect de la différence, de l'Autre, un respect que nous affirmons timidement dans la littérature en service social, mais qui ne semble pas facilement passer le cap des vœux pieux dans nos pratiques. Car, reconnaitre la différence et la respecter réellement voudrait dire un changement majeur dans nos façons d'établir des diagnostics et des plans de traitement des individus marginalisés.

Le service social à caractère spirituel pourrait alors, plutôt que de proposer des façons de voir et de faire basées sur une expertise médicale ou psychologique, expliquer et même défendre, vis-àvis des autres professions, telle attitude, tel comportement, telle divergence d'opinions, voire tel acte considéré comme subversif. Nous pourrions aussi laisser la personne (appelée souvent cliente ou patiente) tout simplement vivre, s'exprimer. Si nous nous inspirions de Jane Addams, nous constaterions que le problème ne se situe pas sur le plan de la personne qui vit dans sa peau les contradictions de notre société marchande. Et si nous adoptions son approche, celle de Jean Vanier ou de Gandhi, nous nous porterions à la défense de ces personnes démunies, car, en voyant avec les yeux du cœur, nous pourrions compatir avec leur vécu, comprendre leur point de vue, les valoriser dans leur expression.

Sri Chinmoy (2002: 242), maitre spirituel qui médite aux Nations Unies avec le personnel et les délégués depuis les années 70 , propose justement cette voie du cœur dans ses enseignements. Il s'agit tout simplement de voir non avec notre logique, mais avec notre cœur, apprendre à voir l'autre comme ayant un rôle essentiel sur terre. Cette démarche fait basculer plusieurs théories et pratiques à caractère mental, intellectuel, parce que de telle sorte, les formes de traitement que notre profession privilégie actuellement ne tiennent plus. C'est le cœur qui compte, avec des valeurs de transcendance, de compassion, d'unité.

\section{Troisième principe : « Médecin, guéris-toi toi-même »}

Cette invitation à l'autoguérison, vieille de quelques millénaires et attribuée à Jésus-Christ lui-même (Luc 4, 20-30), est en train 
"...les théories établissant le pouvoir du cour comme centre de guérison ne font que poindre, mais déjà elles sont en train de revaloriser diverses composantes souvent négligées de l'aide... et de révolutionner l'univers thérapeutique. " de retrouver sa place dans ce débat entre les sciences qui veulent garder le contrôle sur la guérison des gens et les autres approches qui prônent un retour sur soi, sur ses ressources, sur son potentiel intérieur :

There are so many choices in our health care system today that it is hard to know what to do. Having choices is a great gift, but it also brings additional stress. And the more we depend on remedies outside ourselves to solve the problem, the greater our stress becomes. This burden, however, is significantly lessened when we learn the secrets of how to heal ourselves. As we learn to awaken within ourselves the natural self-healing ability, we no longer feel confused about which health program is right for us. We gain confidence in our ability to heal ourselves (John Gray : ix).

Cette citation propose certaines des composantes d'un renouvellement des pratiques sociales basées sur le pouvoir intérieur: l'éveil du Soi, la réalisation de notre potentiel de croissance, à condition que nous cessions de mettre notre confiance en l'autre comme expert et responsable de notre bienêtre, de notre destinée. "We are all truly unlimited, renchérit Sri Chinmoy; if only we dare to try and have faith.»

Plusieurs auteurs ont récemment abordé ce concept de l'autoguérison, un pouvoir intérieur dont nous disposons (Servan-Schreiber, Chadwick), mais qui est encore peu compris. En fait, les théories établissant le pouvoir du cœur comme centre de guérison ne font que poindre, mais déjà elles sont en train de revaloriser diverses composantes souvent négligées de l'aide (télépathie, entraide, cothérapie, etc.) et de révolutionner l'univers thérapeutique. Certaines recherches mentionnent l'intelligence du cœur et étudient les liens dynamiques entre nos deux intelligences (Servan-Schreiber). Ces liens interactifs entre ces deux intelligences se manifestent dans des moments intenses tels l'allaitement, les relations amoureuses ou les rêves. 
«En reconnaissant ce pouvoir intérieur, nous n'aurions pas besoin de recourir à l'autre, sous forme de médicament, de thérapie, etc., pour nous aider. "

«...les sociétés traditionnelles utilisent beaucoup plus le silence comme forme d'expression, de communication et de guérison. »
The strongest relationship between the heart and the emotional brain is a diffuse two-way communication network known as the autonomic peripheral nervous system; this is part of the nervous system that, beyond our conscious control, regulates the functioning of our organs (Servan-Schreiber : 37).

En reconnaissant ce pouvoir intérieur, nous n'aurions pas besoin de recourir à l'autre, sous forme de médicament, de thérapie, etc., pour nous aider. Les concepts de l'empowerment pourraient prendre ici un tout autre sens.

\section{Quatrième principe : au lieu de la parole, le pouvoir du silence}

"In our truest selves, we are after all beings of Silence and Light " Richard Gere

Dans une profession basée sur la parole, sur le dit et l'écrit, valoriser le silence constitue tout un défi. Alors que la spiritualité est essentiellement non-parole, centrée sur l'intérieur, notre profession a misé sur l'analyse des mots, des concepts, des cris exprimés par des personnes souffrantes dans leurs démarches d'aide. Par contre, les sociétés traditionnelles utilisent beaucoup plus le silence comme forme d'expression, de communication et de guérison. Justement parce que sociétés orales, elles n'ont pas eu recours à l'écriture pour s'exprimer, pour s'affirmer. On n'a qu'à penser aux parents inuits : dans leurs traditions, pour élever leurs enfants, ils ne leur parlent pas. Les enfants apprennent par le biais de l'exemple, en silence. Par le même phénomène, c'est par le biais du silence que la spiritualité se vit, s'exprime, s'apprend. La marche, la retraite, la nature, les touchers thérapeutiques, sont quelques manifestations de ce pouvoir du silence. Difficiles arrimages ici entre expressions différentes, voire opposées, à la base desquelles souscrivent de nombreuses thérapies alternatives d'une part et pratiques de spiritualité vivante de l'autre.

Before my soul became my counsel, I was dull, and weak of hearing, reflecting only upon the tumult and 
the cry. But, now, I can listen to silence with serenity and can hear in the silence the hymns of ages chanting exaltation to the sky and revealing the secrets of eternity (Khalil Gibran).

Il faut reconnaitre par ailleurs que les Autochtones ont, sur ce plan, une énorme longueur d'avance sur les sociétés occidentales. En effet, la spiritualité a toujours été pour eux au cœur de l'intervention sociale (Beatch et Stewart 2002). Il en est de même pour les Maoris en Nouvelle-Zélande, les Whanaus en Australie (Nash 148). Pour eux, le silence ne pose pas problème, tout au contraire; ils sont proches de la nature qu'ils qualifient de Mère, et de ses diverses manifestations à travers la Terre.

\section{Cinquième principe : pardon, réconciliation et spiritualité}

"Celui qui pardonne nettoie et parfume son propre coeur »

$$
\text { (Paolo Coelho, Maktub : 63). }
$$

"Le pardon est une valeur de plus en plus explorée dans les pratiques à caractère spirituel. »
Le mot grec pour pardon, asphesis, signifie libération, ouverture, bris des chaines (Vanier : 135). Le pardon est une valeur de plus en plus explorée dans les pratiques à caractère spirituel. Plutôt que d'exiger justice ou revanche par exemple, le pardon se base sur des valeurs de transcendance et de compassion. Divers auteurs abordant ce concept (Jampolsky 1999,Vanier 1998) y voient même plusieurs avantages dans le domaine de la thérapie. La libération des charges émotionnelles reliées au passé, du poids de traumatismes (violence, guerre, agressions, etc.), le fait de pouvoir continuer sa vie en se basant sur le présent et le futur plutôt que sur le passé, telles sont certaines des valeurs qui se dégagent de la littérature sur le pardon. JeanVanier, fondateur de l'Arche, insiste sur la libération qui accompagne le pardon. Pardonner, c'est se débarrasser des sentiments de peine, d'impuissance, de vengeance pour faire place à l'espoir, basé sur le présent et sur notre potentiel. De l'agressivité à la sérénité, de l'indifférence à l'amour, de la haine à la compassion, voici certains des revirements que le pardon peut enclencher. Qui 
"Plusieurs théories centrées sur la justice, l'équité, la revendication s'opposent aux démarches de pardon. D'autres, par ailleurs, voient dans le pardon un acte héroïque, rempli de magnanimité, de courage. " plus est, les approches de réconciliation qu'il propose n'affectent pas seulement la victime, mais par un effet de contagion, peuvent toucher les cœurs des oppresseurs (Vanier : 157).

Un des débats importants au sein des professions d'aide se situe justement autour du concept de pardon. Certains experts critiquent vertement une telle démarche comme constituant une façon de se soumettre à des forces ou à des personnes qui ont abusé de nous. Plusieurs théories centrées sur la justice, l'équité, la revendication s'opposent aux démarches de pardon. D'autres, par ailleurs, voient dans le pardon un acte héroïque, rempli de magnanimité, de courage.

"Forgiveness is the quality of the brave and not of the coward; forgiveness is the attribute of the strong ", suggère Gandhi. Le pardon ne doit pas servir d'excuse pour que l'agresseur continue de perpétrer des actes de violence; bien au contraire, le pardon signifie un cheminement, un respect de soi, dans la dignité. Il ne signifie donc pas l'acceptation de comportements opprimants à notre endroit ou à l'égard d'autres personnes ou cultures. Cependant, reconnaissant que nous avons aussi nos torts, nos limites, nos formes de violence, nous sommes plus prêts à considérer avec un regard spirituel les problèmes ou faiblesses des autres.

À peu près toutes les approches basées sur la spiritualité parlent de pardon et encouragent cette pratique. Kim Phuc, Nelson Mandela, Mahatma Gandhi, font l'éloge de cette façon de faire qui implique un dépassement de soi et reflète des valeurs de noblesse d'âme que la psychothérapie traditionnelle met de côté, pour ne pas dire qu'elle regarde avec mépris. Par exemple, depuis la fin de l'apartheid, l'Afrique du Sud a vu une montée de cette tendance à pardonner. Lors de l'indépendance du pays, Nelson Mandela a rappelé aux Noirs qu'il faut maintenant vivre, coexister, avec la population blanche qui les a opprimés. Et pour vivre en communauté, il a mis en place des façons de faire basées sur le pacifisme et non sur la vengeance. Autre exemple : Gandhi a prôné l'exemple de la non-violence face à des agresseurs qui ont blessé, massacré, tué des milliers de ses compatriotes. Et il est parvenu à ses fins. «Le pardon, c'est la qualité des personnes braves et non des lâches. Le pardon, c'est la caractéristique des personnes fortes ", 
"Le pardon signifie la liberté de l'esprit, la félicité du cour et l'unité de l'âme»

"...le pardon et la réconciliation constituent des dimensions qui suggèrent certaines différences de fond entre les approches progressistes d'intervention et les approches spirituelles de l'aide." écrivait-il. "Le pardon signifie la liberté de l'esprit, la félicité du cœur et l'unité de l'âme ", réplique Sri Chinmoy. Plusieurs conflits interculturels ou interraciaux qui ont cours pourraient explorer le pardon comme approche alternative à la violence et à la vengeance qui ont cours actuellement.

Il va sans dire que de telles approches sont remises en question, même par les tenants les plus progressistes de l'intervention psychologique et sociale, qu'elle soit structurelle, féministe ou radicale. À ce titre, le pardon et la réconciliation constituent des dimensions qui suggèrent certaines différences de fond entre les approches progressistes d'intervention et les approches spirituelles de l'aide.

Dans le Mémorial de Caen, en Normandie, dont la devise est justement "La douleur m'a brisé, la fraternité m'a relevé, de ma blessure a jailli un fleuve de paix ", une grande salle est consacrée à celles et à ceux qui, dans l'histoire de l'humanité, ont prôné et prônent encore des approches pacifistes, basées sur le pardon et la réconciliation. Plusieurs espaces muséographiques démontrent que les artisans de paix, d'harmonie, de réconciliation, se retrouvent davantage dans les sociétés traditionnelles que les sociétés modernes, dans les cultures orales plutôt que dans les cultures où l'écriture prédomine, et dans les cultures orientales plutôt qu'occidentales. De nombreux exemples illustrent diverses traditions et pratiques pacifistes des peuples dits primitifs : la roue médicinale, le calumet de paix et de réconciliation, qu'on fume à tour de rôle jusqu'à ce qu'un consensus soit atteint, le shir, sage nommé par plusieurs clans, en Somalie, les mouvements de paix Shanti ou AHIMSA et la pratique de la diversité spirituelle Sangha, entre autres exemples.

Toutes ces pratiques privilégient la non-violence pour contrer la violence et l'agression. Selon cette perspective, la contribution de l'Occident à l'évolution de l'humanité aura-t-elle davantage été de développer des armes de destruction, et de proposer, voire d'imposer, des formes violentes de lutte à la violence, phénomène d'où résulte, nous le constatons tous actuellement, l'escalade de la violence? "We are going to have peace, even if we have to fight for it " proclamait Eisenhower. À l'opposé de cette vision 
militaire de l'harmonie, le Mémorial de Caen rappelle que des quatre grands leaders de la paix au XX $\mathrm{XX}^{\mathrm{e}}$ siècle, aucun n'est blanc ou occidental (Mahatma Gandhi, Nelson Mandela, Martin Luther King et Desmond Tutu $)^{4}$.

\section{Impact pour l'intervention : une façon autre de voir l'Autre}

"Cette distance que nous avons créée entre le matériel et le spirituel, nous n'avons plus le choix que de la réduire et éventuellement de l'éliminer." »

"...force est de constater que nous avons perdu beaucoup, entre autres sur le plan spirituel, et que nous avons fait perdre beaucoup aux cultures opprimées. »
Cette distance que nous avons créée entre le matériel et le spirituel, nous n'avons plus le choix que de la réduire et éventuellement de l'éliminer ${ }^{5}$. Pour poursuivre nos réflexions, pour repenser nos pratiques, il me semble important de souligner quelques directions possibles qui puissent alimenter nos réflexions et nos interventions.

Piste 1 - Apprendre de nos erreurs : le rôle exemplaire des personnes et des peuples opprimés

La colonisation a pratiquement détruit, consciemment ou non, la dimension sacrée existant au coeur des sociétés traditionnelles, qu'elles soient amérindiennes en Amérique, maories en NouvelleZélande, tout autant que celles des cultures celtique, inuite, tsigane ou autres. Ces liens avec le sacré, ces liens entre l'humain, la Mère Terre et l'Univers, cette harmonie tant chantée a fait place à des comportements de domination, basés sur une attitude de supériorité de la part des colonisateurs et de l'Occident vis-à-vis des cultures et des peuples qualifiés de primitifs. De la sorte, nous, de l'ère industrielle et capitaliste, avons exploité la terre sans tenir compte de l'écologie, de l'histoire, de la culture, de la spiritualité. Après quelques siècles d'exploitation sauvage des ressources et une utilisation tout aussi honteuse des personnes comme outils de développement, force est de constater que nous avons perdu beaucoup, entre autres sur le plan spirituel, et que nous avons fait perdre beaucoup aux cultures opprimées. Mais ces peuples blessés restent là et, encore une fois, nous tendent la main, nous invitant 
"Les gens spirituels opteront pour des approches pacifistes, harmonieuses, même dans les moments de grande tension.

... apprendre de nos erreurs peut vouloir dire de demander pardon aux citoyens autochtones pour les écoles résidentielles ou pour les milliers d'enfants que nous avons placés dans des foyers blancs,..." à ne pas rater le prochain tournant, celui qui nous permettrait d'apprendre de nos erreurs, grâce à des héros et héroïnes comme Nelson Mandela, Kim Phuc et des milliers d'autres.

En tentant d'appliquer cela à l'oppression des Amérindiens, Mary Nash propose que les peuples autochtones, opprimés par 500 ans de colonisation, plutôt que de se centrer sur la haine et de chercher vengeance, guident l'homme blanc, montrent à l'oppresseur comment regarder le monde de façon différente, voir le sacré dans la création, voir le Créateur dans son œuvre. "Aboriginal peoples might rather guide the white man to look differently at the world, to see the sacred in creation and to value it above material wealth " (Nash : 137).

Le même principe s'applique à beaucoup d'autres aspects de l'oppression, à diverses instances d'une intervention à caractère spirituel, que ce soit sur le plan des classes sociales, ou sur ceux des sexes et des cultures. Nous avons le choix entre une approche de vengeance et une approche de compassion, entre exiger la justice ou pratiquer le pardon. Les gens spirituels opteront pour des approches pacifistes, harmonieuses, même dans les moments de grande tension.

À titre d'exemple, apprendre de nos erreurs peut vouloir dire de demander pardon aux citoyens autochtones pour les écoles résidentielles ou pour les milliers d'enfants que nous avons placés dans des foyers blancs, ou encore demander pardon aux personnes psychiatrisées pour les avoir internées parce qu'elles pensaient autrement, aux enfants de Duplessis pour toutes ces vies perdues, pour des diagnostics aussi faux que nécessaires à la survie des institutions qu'on voulait à tout prix sauvegarder. Cela peut aussi vouloir dire pardonner aux missionnaires qui nous ont prêché un Évangile rempli de contradictions ou à la personne qui nous a violenté, personnellement ou dans notre culture.

Sur un plan personnel, c'est seulement après que j'eus pardonné à deux prêtres qui ont pratiquement détruit ma jeunesse, que j'ai pu retrouver la paix intérieure. Lorsque je suis allé rencontrer celui qui est encore vivant,j'ai pu me libérer, non par la vengeance, mais grâce au pardon. En fait, j'ai alors réalisé qu'il est beaucoup plus 
"À ce chapitre, les psychothérapies à visée humaniste ou transpersonnelle n'ont pas une approche aussi exigeante ni aussi noble que la démarche spirituelle. » difficile - mais combien plus noble - de pardonner que de me venger ou d'exiger compensation, comme certains m'incitaient à le faire. Ce pardon m'a libéré de cette souffrance qu'aucune amende ou punition n'aurait réussi à compenser. À ce chapitre, les psychothérapies à visée humaniste ou transpersonnelle n'ont pas une approche aussi exigeante ni aussi noble que la démarche spirituelle.

\section{Piste 2 - L'héliomonie}

Elle est la transmission des enseignements de sagesse basée sur les traditions initiatiques. Cette synthèse nous ramène progressivement au centre, c'est-à-dire au Soi. Ainsi, nous pouvons commencer à mieux rayonner ce que nous sommes vraiment, notre vraie nature. L'héliomonie n'est ni un dogme ni une religion, elle est la Vie que l'on choisit parce qu'elle est authentique, simple, créatrice et joyeuse. Elle invite à un vrai travail sur soi pour transformer et transmuter ce qui ne sert pas notre croissance, pour notre plus grand bien et celui des autres. Ces lois sont celles qui régissent la nature et toutes créations : les comprendre n'est pas une tâche intellectuelle, mais bien une intégration par le vécu et le ressenti, c'est-à-dire par les enseignements de la Vie. Lorsque ceux-ci sont compris et respectés, la vie s'offre comme le plus beau des cadeaux, dans toute sa splendeur (http://www.french.dhamma. org/index.html).

\section{Piste 3 - La technologie intérieure}

Braden (2000) suggère que l'émotion est le commutateur qui déclenche des codes d'ADN précis dans notre corps. Ce sont les mêmes codes qui nous donnent la liberté de vivre sans maladie ni détérioration en avançant dans le temps linéaire. Il rapporte que des chercheurs ont récemment démontré au monde occidental un phénomène enseigné dans les écoles de mystères depuis des millénaires à savoir que l'émotion humaine détermine la structure de l'ADN dans le corps. En outre, des expériences de laboratoire ont montré que l'ADN définit de quelle façon des patterns de lumière, exprimés sous forme de matière, encerclent le corps humain. Autrement dit, les chercheurs ont découvert 
"...la science a démontré que l'ADN est directement relié à notre capacité de pardonner et d'aimer. ...la technologie qui sous-tend l'amour et le pardon est une science aussi ancienne qu'universelle appelée aujourd'hui compassion, qui d'ailleurs est le noyau de notre nature véritable. » que la disposition de l'énergie (atomes, bactéries, virus, climat, et même les gens) qui entoure notre corps est directement reliée aux sentiments et aux émotions qui émanent de ce dernier.

Il rapporte également que la science a démontré que l'ADN est directement relié à notre capacité de pardonner et d'aimer. Dans cette optique, la science de l'amour, du pardon et du lâcherprise n'a rien de nouveau et est basée sur notre vraie nature. Il ajoute que la technologie qui sous-tend l'amour et le pardon est une science aussi ancienne qu'universelle appelée aujourd'hui compassion, qui d'ailleurs est le noyau de notre nature véritable.

\section{Piste 4 - La paix intérieure : la méditation}

Vispassana est l'une des techniques de méditation les plus anciennes de l'Inde. Perdue pendant longtemps, elle fut redécouverte il y a 2500 ans par Gautama le Boudha. Vipassana veut dire "voir les choses telles qu'elles sont en réalité ». C'est le processus d'autopurification par l'observation de soi. On commence par observer la respiration normale et naturelle afin de concentrer l'esprit. Une fois l'attention aiguisée, on procède à l'observation de la nature changeante du corps et de l'esprit, faisant ainsi l'expérience des vérités universelles de l'impermanence, de la souffrance et du non-soi. C'est cette prise de conscience par expérience directe qui constitue le travail de purification. Le chemin dans son ensemble, appelé Dhamma, constitue un remède universel à des problèmes universels et il ne s'apparente en aucune façon à une religion établie. Donc, il peut être pratiqué librement, sans distinction de race, de milieu ou de religion, partout et en tout moment.

La méditation vise les plus hauts objectifs spirituels : la libération totale et l'éveil de l'Être. Son but n'est jamais la guérison de maladies physiques, mais la purification mentale a comme effet secondaire d'éliminer de nombreuses maladies psychosomatiques. En fait, la méditation dissout les trois causes de toutes nos souffrances: l'avidité, l'aversion et l'ignorance. Grâce à une pratique suivie, la méditation relâche les tensions développées dans la vie quotidienne et délie les nœuds créés par 
l'ancienne habitude de réagir de manière excessive aux situations agréables ou désagréables. Un nombre incalculable de pratiques ou techniques de méditation existent de nos jours; on aurait avantage à les intégrer à nos pratiques quotidiennes; elles aideraient à prévenir des maladies de civilisation telles que le burn-out, la dépression ou l'angoisse.

\section{Conclusion : transformations radicales ou cosmétiques?}

"Ces percées ont eu peine à se concrétiser, devant lutter à la fois contre l'establishment religieux et l'économie de marché, dont les valeurs éthiques et les principes moraux sont fort distants de toute préoccupation spirituelle."
Notre conception du monde nous a conduits à bâtir nos institutions et c'est d'elles que nous sommes maintenant prisonniers. Le progrès, pour nous les riches, c'est la croissance de ces institutions (Illich : 155).

Au cours de l'histoire récente, alors que les dimensions intérieures de l'être humain étaient reléguées à la religion, les dimensions extérieures et quantifiables ont été prises en charge et exploitées par la science et la raison. D'où une division à peu près hermétique qui s'est créée entre les deux, et, de surcroît, une confusion, en bien des endroits, entre religion et spiritualité.

Dès lors, l'histoire récente témoigne d'un développement religieux basé sur plusieurs valeurs et principes, généralement plus conservateurs que progressistes, et quelques percées de développement basées sur le spirituel ou le sacré. Ces percées ont eu peine à se concrétiser, devant lutter à la fois contre l'establishment religieux et l'économie de marché, dont les valeurs éthiques et les principes moraux sont fort distants de toute préoccupation spirituelle.

Qui plus est, les mouvements progressistes en sciences sociales, tant sur le plan de l'égalité des sexes, des classes sociales, des cultures, des régions, des races, que des préoccupations écologiques, ont souvent été étouffés par les élites bourgeoises, qu'elles soient religieuses ou capitalistes. Dans ce contexte, les 
"Sous couvert de développement et d'épanouissement personnel, la personne moderne s'est coupée de nombreuses réalités; "

\section{"La spiritualité} se reflète de multiples façons, mais généralement ce sont des valeurs d'être plutôt que des valeurs d'avoir qui la caractérisent. » approches de conscientisation qu'on a vu poindre dans les années 1970, en particulier, celles fondées sur les enseignements de Paolo Friere, résistent très difficilement aux rouleaux compresseurs de professions d'aide vouées à maintenir des préoccupations d'ordre individuel et intrapersonnel. Sous couvert de développement et d'épanouissement personnel, la personne moderne s'est coupée de nombreuses réalités; sa relation à son corps, considéré presque comme un objet mécanique aux multiples morceaux juxtaposés, sa vie spirituelle, où il a ignoré le pouvoir des chakras, de la transcendance, de la télépathie, des chamans, son rapport à l'Absolu ont été mis de côté au profit de considérations mercantiles.

L'intérêt grandissant pour la spiritualité vient-il ajouter une dimension nouvelle à l'intervention sociale, ou plutôt nous oblige-t-il à repenser les bases idéologiques de notre profession et nos approches thérapeutiques? Toute action est spirituelle; l'intervention ne peut être que spirituelle, car elle est au cœur même de la vie. La spiritualité se reflète de multiples façons, mais généralement ce sont des valeurs d'être plutôt que des valeurs d'avoir qui la caractérisent.

En regard de ce qui a été présenté, d'aucuns seraient peutêtre tentés de suggérer que la dimension spirituelle peut tout simplement s'ajouter aux autres composantes de l'aide. Ainsi, elle viendrait compléter une mosaïque d'éléments constituant une aide véritable. En fait, j'estime qu'il s'agit plutôt de remettre en question les présupposés actuels de l'aide, fondés sur des assises psychologiques, médicales ou bio-psycho-sociales, à la Richmond ou à la Freud, et de les remplacer par une conception de l'aide qui s'articule sur des registres qui remettent en question la relation d'aide et ses rapports d'expertise, de pouvoir, de savoir. Être — ou devenir - des êtres spirituels signifie un engagement vis-à-vis des valeurs de changements sociaux basés sur l'équité,l'harmonie, certes, mais également sur une conscience intérieure et une conscience supérieure, essentielles à l'articulation et la réalisation de ces changements. 


\section{Bibliographie}

ABOT, Charles (1994). Mutation, Troisième millénaire, Paris, Maromme.

AUGER, Lucien (1986). La démarche émotivo-relationnelle en psychothérapie et relation d'aide, Montréal, Éditions Ville-Marie.

BEATCH, Romeo and Bruce Stewart (2002). "Integrating Western and Aboriginal Healing Practices”, in: Spirituality and Social Care, Philadelphia, Mash et Stewart, Jessica Kingsley publ.

BOIRON, Christian (1993). Et si nous pensions autrement la vie? Paris, Albin Michel.

BOURGAULT, Luc (1985). L'héritage sacré des peuples amérindiens, Ottawa, Édition de Mortagne.

BRADEN, Gregg (2000). Marcher entre les mondes : La science de la compassion, Outremont, Ariane.

BRETON, Margot, (2002). "Empowerment Practice in Canada and the United States: Restoring Policy Issues at the Center of Social Work", The Social Policy Journal 1, (1), 19-34.

BROWN, Joseph (1982). The Spiritual Legacy of the American Indian, New York, Crossroad Publishing.

CANDA, E.R. (1988). “Spirituality, Religious Diversity and Social Work Practice”, Social Casework, 69(4), avril, 238-247.

CARKHUFF, Robert (1988). L'art d'aider, Montréal, Éditions de l'homme.

CARKHUFF, Robert et Bernard Berenson (1977). Beyond Councelling and Therapy, New York, Holt, Rinehart and Winston.

CASTANEDA, Carlos (1984). L'herbe du diable et la petite fumée, Paris, C. Bourgeois.

CHADWICK, Gloria (1995). Spirituality and Self-Empowerment, Chicago, Contemporary Books.

COELHO, Paulo (2004). Maktub, Paris, Éditions Anne Carrière.

COQUIN, Pierre (1997). "L'évolution, de la genèse à notre temps ", $3^{e}$ millénaire, no. 27, 48-72.

DUFORT, F. et Guay J. (éds) (2001). Agir au cour des communautés : la psychologie communautaire et le changement social, Ste-Foy, Les Presses de l'Université Laval.

FREIRE, Paolo (1974). Pédagogie des opprimés, Paris, Masparo.

GABOURY, Placide (1993). Vivre imparfait, Éditions Mortagne.

GRAY, John (2002). "Forward to: Zhi Gang Sha”, Power Healing, San Francisco, Harper.

ILLICH, Ivan (1971). Libérer l'avenir, Paris, Point.

JAMPOILSKY, Gerald, (1999). Forgiveness, the Greatest Healer of All, Beyond Words Publishing, Hillsboro.

JUNG, Gustav (1964). L'homme et ses symboles, Paris, Robert Laffont.

JUNG, Gustav (1976). Problèmes de l'âme moderne, Paris, Buchet/Chastel.

JUNG, Gustav (1998). L'homme à la découverte de son âme, Paris, Albin Michel.

KRIPPNER, Stanley et Patrick Welch (1994). Spiritual Dimensions of Healing, New York, Irvingston Publ.

LEMIEUX, Raymond (2000). Misère de la religion, grandeur du spirituel, texte présenté lors du congrès Événement 2000, Le bilan de santé du spirituel et du religieux, Montréal.

MCKOY, Kelly (2005). Saint Jane: Religion and the Social Ethic of Jane Addams, http://www. ptloma.edu/HistPolSci/Students/Kelli_McCoy.htm (Site consulté en juillet 2005).

MULLALY, Robert (1997). Structural Social Work: Ideology, Theory and Practice, Toronto, Oxford University Press. 
MYSS, Caroline (1996). Anatomy of the Spirit: The Seven Stages of Power and Healing, New York, Random House.

NASH, Mary (2002). "Spirituality and Social Work in a Culturally Appropriate Curriculum”, in: Mary Nash, Spirituality and Social Care, Jessica Kingsley Publishers, Philadelphia, 129-149.

OUELLETTE, Brian (2005). Marrying the Head and the Soul, Site consulté en juillet 2005 : http:// www.stthomasu.ca/publications/teaching/fall2000/ouellette.htm.

PLATTEAU, Jean-Philippe (1986). "Fonction euphémisante et mystificatrice de l'aide », Dis-moi qui tu aides, Presses universitaires de Namur, Namur, 183-218.

ST-AMAND, Nérée (2003). "Interventions opprimantes ou conscientisantes ", Reflets, revue ontaroise d'intervention sociale et communautaire, Automne, 9:2, 139-162.

ST-AMAND, Nérée (2001). "Dans l'ailleurs et l'autrement : pratiques alternatives et service social », Reflets, revue ontaroise d'intervention sociale et communautaire, 7:2, 30-74.

SERVAN-SCHREIBER, David (2004). The Instinct to Heal, New York, Rodale.

SIVARASKA, Sulak (1992). Seeds of Peace: A Buddhist Vision for Renewing Society, Parallax Press, Berkley.

SPINK, Kathryn (1990). Jean Vanier and L'Arche: A Communion of Love, London, Darton, Longman and Todd.

SRI AUROBINDO (1987). L'idéal de l'esprit humain, Paris, Buchet/Chastel.

Sri Aurobindo (1994). Le cycle humain, Paris, Buchet/Chastel.

SRI CHINMOY 1972. Eastern Light for the Western Mind, Sri Chinmoy's University Talks, New York, AUM Publications.

SRI CHINMOY (1992). L'enseignement du silence, Paris, Éditions Sri Chinmoy.

THE SACRED TREE (1989). Reflections on Native American Spirituality, Twin Lakes, Lotus Light Publications.

\section{Sites Internet : Sites consultés en juillet-août 2005}

Addams, Jane: site Hull-House Highlights http://www.uic.edu/jaddams/hull/hull_house.html Art thérapie :

1- http://www.reseauproteus.net/therapies/artther/

2- http://www.wholehealthmd.com/refshelf/substances_view/1,1525,671,00.htm

Auroville, cité de l'harmonie universelle : http://www.auroville.org/

Co-councelling : http://co-cornucopia.org.uk/index.htm

Einstein:

1- http://www.quotationspage.com/quotes/Albert_Einstein/

2- http://www.heartquotes.net/consciousness.html

Freud, Sigmund :

1- http://pages.globetrotter.net/desgros/freud/

2- http://pages.globetrotter.net/desgros/freud/1856-1885.html

Gandhi, Mahatma: http://www.spiritusmundi.net/personnalites/gandhi.htm

Héliomonie (Royaume de Shamballah) : http://heliomonisme.com/fr/htm/messages.htm

Jung, Carl : http://www.ship.edu/ cgboeree/jung.html\#CARL\%20JUNG 
Martin Luther King :

1- J'ai un rêve... http://perso.wanadoo.fr/felina/doc/decl/luther_king.htm

2- I have a dream... http://www.mecca.org/ crights/dream.html

Méditation Vipassana : http://www.french.dhamma.org/index.html

Non-violence : http://forget-me.net/nonviolence/index.php

Parlement des religions du monde : http://www.cpwr.org/2004Parliament/

Patch Adams Gesundheit Institute: http://patchadams.org

Peace Pilgrim :

1- http://www.peacepilgrim.net/

2- http://www.forget-me.net/PeacePilgrim/ (français)

Ressources alternatives : http://www.reseauproteus.net/proteus.htm

St-Amand, Nérée: http://aix1.uottawa.ca/ nstaman/alternatives/Alternatives.htm

Spiritualité autochtone : http://www.reseauproteus.net/therapies/artther/

Sri Chinmoy: www.srichinmoy.org

Vanier, Jean :

1- http://www.larchecanada.org

2- http://perso.wanadoo.fr/archevallee/FrAdlv/fvanier.html

Wollstonecraft, Mary: http://www.spartacus.schoolnet.co.uk/Wwollstonecraft.htm

\section{Notes}

1 À titre d'exemple, dans la région d'Ottawa, on n'a qu'à consulter le mensuel Tone, ou son équivalent, Cheminement, dans l'Outaouais québécois, pour constater que foisonnent les forums, conférences, séminaires, sur diverses dimensions et manifestations de la spiritualité.

2 Les enfants de Duplessis, Mount Castle, et les Écoles résidentielles en sont quelques exemples au Canada, sans parler des abus physiques, psychologiques ou sexuels des membres des congrégations religieuses ou de son clergé un peu partout en Occident.

3 Il serait long de nommer les nombreux auteurs auxquels je me réfere ici. Qu'on pense simplement au fait que les grandes revues d'intervention sociale, de service social, n'abordent à peu près jamais cette dimension dans leurs écrits (Service social, Intervention sociale, Nouvelles pratiques sociales, pour ne nommer que quelques-unes au Québec) et les auteurs qui ont marqué le service social psychologisant, en particulier Charkhuff, Berenson, Truax, Richmond et Auger.

4 Ce faisant, il passe quand même sous silence l'immense contribution d'autres personnes, femmes en particulier, que l'histoire officielle a peu retenues mais qui ont joué un rôle marquant dans cette quête d'harmonie (qu'on pense à Peace Pilgrim ou à Mary Wollstonecraft, par exemple).

5 Dans cette optique, j'ai déjà créé un site Internet portant justement sur les approches alternatives qui affectent le service social et desquels nous pourrions nous inspirer pour voir autrement, faire autrement, adopter des principes de vie et des approches d'intervention http://aix1.uottawa. $\mathrm{ca} / \sim$ nstaman/alterna-tives/Alternatives.htm 